\title{
BMJ Open Indications and outcomes of paediatric tracheotomy: a descriptive study using a Japanese claims database
}

\author{
Kayoko Mizuno, ${ }^{1,2}$ Masato Takeuchi, ${ }^{2}$ Yo Kishimoto, ${ }^{1}$ Koji Kawakami (D) ,2 \\ Koichi Omori $^{1}$
}

To cite: Mizuno K, Takeuchi M Kishimoto Y, et al. Indications and outcomes of paediatric tracheotomy: a descriptive study using a Japanese claims database. BMJ Open 2019;9:e031816. doi:10.1136 bmjopen-2019-031816

- Prepublication history for this paper is available online. To view these files, please visit the journal online (http://dx.doi org/10.1136/bmjopen-2019031816).

Received 22 May 2019 Revised 22 November 2019 Accepted 02 December 2019

A Check for updates

C Author(s) (or their employer(s)) 2019. Re-use permitted under CC BY-NC. No commercial re-use. See rights and permissions. Published by BMJ.

${ }^{1}$ Department of OtolaryngologyHead and Neck Surgery, Kyoto University Graduate School of Medicine Faculty of Medicine, Kyoto, Japan

${ }^{2}$ Department of

Pharmacoepidemiology, Kyoto University Graduate School of Medicine Department of Public Health, Kyoto, Japan

Correspondence to Dr Koji Kawakami; kawakami.koji.4e@kyoto-u. ac.jp

\section{ABSTRACT}

Objective To examine the incidence of and indications for paediatric tracheotomy to clarify the disease burden relevant to tracheotomy in a population-based context. Design A descriptive analysis of a retrospective cohort. Setting This study utilised a nationwide claims database in Japan constructed by JMDC (Tokyo, Japan). The database includes claims data for approximately 3.75 million insured persons (approximately $3.1 \%$ of the population of Japan) comprising mainly company employees and their family members.

Participants We identified children registered to have undergone tracheotomy from 2005 to 2017 among about 1.2 million children aged $0-15$ years.

Main outcome measures The characteristics of the study population, and indications for tracheotomy, duration of hospital stay, duration of mechanical ventilation, duration of tracheotomy dependence, complications related to tracheotomy and death were assessed. When there were multiple indications, classification for a child into multiple groups was allowed.

Results The study included 215 children (120 males, $56 \%)$. The median age at tracheotomy was 0.8 years. The most common age at tracheotomy was less than 12 months ( $n=127,59.1 \%)$. The most common indications for tracheotomy were chronic lung disease $(n=79$, $36.7 \%)$, followed by neuromuscular disease $(n=77$, $35.8 \%)$, cardiovascular disease $(n=53,24.3 \%)$, upper airway obstruction $(n=43,20 \%)$, premature birth and related conditions $(n=34,15.8 \%)$, trauma $(n=16,7.4 \%)$, prolonged ventilation due to other causes $(n=12,5.6 \%)$ and malignancy $(n=9,4.2 \%)$. The median duration of tracheotomy dependence was 17.2 months. During the follow-up period, decannulation was achieved in 84 children (39.1\%), and the median time from tracheotomy to decannulation was 12.0 months.

Conclusions Most paediatric tracheotomies were performed due to chronic underlying diseases, and the mean duration of tracheotomy dependence was nearly $1-1 / 2$ years. The long-term duration of tracheotomy dependence might have some impacts on patients' physical and mental development and the quality of life.

\section{INTRODUCTION}

The common indications for paediatric tracheotomy have changed from acute causes, such as respiratory tract infections and trauma, to

\section{Strengths and limitations of this study}

This study is the first Asian nationwide study that reported the outcomes of paediatric tracheotomy depending on the indications.

- We used a nationwide insurance claims database that can track data for each patient, even if the patient visited or was hospitalised at multiple medical institutions.

- The severity and details of the indication of tracheotomy could not be determined because of the lack of this type of information in the claims record; the accuracy of coding may be another limitation.

- In some patients, the durations of mechanical ventilation and tracheotomy dependence may have continued after the follow-up period of this study, so we might have underestimated the durations of mechanical ventilation and tracheotomy dependence.

- The cause of death could not be identified from the database.

chronic causes, such as neurological diseases and upper airway abnormalities. These changes have resulted from decreasing infection by introducing vaccines and preventing trauma patients from becoming serious due to advancements in treatment and technology over the past several decades. ${ }^{12}$ Due to the change in indications for paediatric tracheotomy, the duration of tracheotomy dependence has been increasing. ${ }^{12}$

The data regarding paediatric tracheotomy mentioned above are derived from single-centre experiences or from nationwide surveys performed only in Western countries. ${ }^{3-11}$ Contemporarily, large-scale epidemiological data from other regions may expand our knowledge. Some studies have reported that the outcomes of paediatric tracheotomy depend on the indications, ${ }^{568-11}$ but some of these studies are single-institutional studies, and their results could be influenced by specific practices of the institution such as the indication for tracheotomy and the timing of decannulation and discharge. ${ }^{8-11}$ Other 
studies are multi-institutional studies, but the studies used data only during hospitalisation period. ${ }^{5}{ }^{6}$ In chronic diseases, particularly tracheotomy-related outcomes such as decannulation, complication and mortality may occur not only during hospitalisation but also after discharge or at other medical facilities.

In this study, we aimed to examine the incidence of and, indications for paediatric tracheotomy and outcomes, such as the length of hospital stay, weaning from ventilation, complications, decannulation and mortality, using a nationwide claims database that tracks data for each patient, even if the patient visited or was hospitalised at multiple medical institutions in Japan to clarify the disease burden relevant to tracheotomy.

\section{MATERIALS AND METHODS \\ Data source}

This descriptive study utilised a nationwide insurance claims database in Japan constructed by JMDC (Tokyo, Japan). ${ }^{12} \mathrm{JMDC}$ is a commercial data vendor that collects data from $>100$ employer-based insurances. The database has accumulated reimbursement data from approximately 3.75 million insured persons (approximately $3.1 \%$ of the population) mainly comprising company employees and their family members since 2005 . The database contains the following information: patient characteristics (age and sex), medical and pharmacy claims data, clinical diagnoses coded using the International Classification of Diseases 10th revision (ICD-10) and medical procedures coded using Japan-specific standardised procedure codes. The database can track data for each patient in chronological order, even if the patient visited or was hospitalised at multiple medical institutions. The need to obtain individual informed patient consent was waived because this study was a secondary analysis of an anonymous patient database.

\section{Study cohort}

From a database of about 1.2 million children, we extracted data for 215 children aged $0-15$ years who underwent tracheotomy from 1 January 2005, to 31 December 2017. These patients were identified by the Japanese procedure code for tracheotomy (procedure code number: K386). Children who underwent two or more tracheotomies were excluded.

The following clinical data were collected: sex, age at tracheotomy, indications for tracheotomy, duration of hospital stay, duration of mechanical ventilation, duration of tracheotomy dependence, complications related to tracheotomy and death.

In this study, we identified indications for tracheotomy according to the ICD-10 codes for each child. We reviewed the ICD-10 codes both during hospitalisation when the tracheotomy was performed and at outpatient visits before hospitalisation. Indications for tracheotomy were categorised into the following groups: (1) neuromuscular disease (eg, hypoxic brain injury, encephalopathy, congenital anomalies of the central nervous system, muscular dystrophies, hydrocephalus), (2) upper airway obstruction (eg, congenital malformation, infection), (3) cardiovascular disease, (4) premature birth and related conditions, (5) trauma (eg, head injury, upper airway trauma, thoracic injury, burn injury), (6) chronic lung disease (eg, pneumonia, chronic obstructive pulmonary disease, pulmonary hypoplasia), (7) malignancy (eg, brain neoplasm, haematological neoplasm, thyroid neoplasm) and (8) prolonged ventilation due to other causes (eg, gastrointestinal disease, liver disease, metabolic disorder). When there were multiple indications, classification for a child into multiple groups was allowed.

From the claims record, we estimated the duration of mechanical ventilation and the duration of tracheotomy dependence of each patient by calculating the cumulative days under ventilation use at both hospital and home. The duration of mechanical ventilation was determined by the date of additional use or the date a home management fee for mechanical ventilation was added (procedure code numbers: A306, A307, C107, C164, J026, $\mathrm{J} 045$ ), and the duration of tracheotomy dependence was determined by the date a home management fee for tracheotomy (procedure code number: C112) or the date a material cost for an artificial nose for tracheotomy patients was added (procedure code number: C169). The fees, additional use and material cost were added once a month, so the durations of mechanical ventilation and tracheostomy dependence were calculated on a monthly basis. For the duration of mechanical ventilation, a continuous period including the month when tracheostomy was performed was used. The duration of tracheotomy dependence was calculated starting from the month when the tracheotomy was performed. The end of mechanical ventilation management was defined as the month that an additional use or fee was added for the last time and the month after which an additional use was not recorded for more than 3 months. The end of tracheotomy dependence was defined in the same way. The follow-up period of this study was from 1 January 2005 to 31 December 2017.

Complications related to tracheotomy were identified by the diagnosis codes for dysfunction, obstruction, bleeding, infection of a stoma (ICD-10 code number: J950), intratracheal bleeding (ICD-10 code number: R048) and intratracheal granulation (ICD-10 code number: J398). We identified the complications that occurred within all available follow-up data including the index hospitalisation, outpatient visits and the subsequent hospitalisations (if they occurred). We calculated the risk of tracheotomy-related complications of all children included this study. It was difficult to ascertain whether pneumothorax and pneumonia were attributed to tracheotomy or other causes. Thus, in this study, we estimated the locally occurring complications of tracheotomy listed above. 


\section{Statistical analysis}

The characteristics of the study population are summarised using proportions for categorical variables and medians and quantiles (Q1 (the first quantile), Q3 (the third quantile)).

Mann-Whitney U test and Fisher's exact test were used to compare continuous variables and categorical variables, respectively.

We performed univariable logistic regression analyses to evaluate the association between indications for tracheotomy and its outcomes, such as discharge with cannulation, occurrence of decannulation and mortality, and multivariable logistic regression analyses were used to evaluate the association between occurrences of complications related to tracheotomy, and age and duration of tracheotomy dependence. For duration of tracheotomy dependence, we used 2 years as a cut-off to divide the cohort for the analysis because $\mathrm{Ha}$ et al reported that the relative risk of a persistent tracheocutaneous fistula was significantly increased when the duration of tracheostomy dependence was greater than 2 years, ${ }^{2}$ and we assumed that other complications related to tracheotomy would also increase when the duration of tracheotomy dependence was longer than 2 years. The results were expressed as ORs with $95 \%$ CIs.

All statistical tests were two-sided; $p<0.05$ was considered statistically significant. All data analyses were performed using SAS for Windows (V.9.4; SAS Institute).

\section{Patient and public involvement}

Patients and the public were not involved in the study design or the conduct of the study.

\section{RESULTS}

\section{Demographics and clinical characteristics}

From the JMDC database, we identified 215 children (120 males, $56 \%$ ) who underwent tracheotomy from 1 January 2005 to 31 December 2017. Table 1 presents the demographic and clinical characteristics of the study cohort. The median age at tracheotomy was 0.8 years (IQR: $0.3-4.4$ years). Tracheotomies were performed most often when patients were younger than 12 months ( $\mathrm{n}=127,59.1 \%)$. The most common indications for tracheotomy were chronic lung disease $(n=79,36.7 \%)$, followed by neuromuscular disease $(\mathrm{n}=77,35.8 \%)$, cardiovascular disease $(\mathrm{n}=53,24.2 \%)$, upper airway obstruction $(\mathrm{n}=43,20.0 \%)$, premature birth and related conditions $(\mathrm{n}=34,15.8 \%)$, trauma $(\mathrm{n}=16,7.4 \%)$, prolonged ventilation $(\mathrm{n}=12,5.6 \%)$ and malignancy $(\mathrm{n}=9,4.2 \%)$. Ninety-six children $(44.7 \%)$ had two or more indications. Chronic lung disease and neuromuscular disease $(n=31)$ were the most commonly paired indications, followed by chronic lung disease and premature birth. In the upper airway obstruction group, congenital malformation was the most common cause $(\mathrm{n}=38,88.3 \%)$, followed by benign tumours $(\mathrm{n}=3)$ and infection $(n=2)$. The median duration of tracheotomy dependence of all children included this study was 17.2
Table 1 Clinical characteristics of paediatric patients undergoing tracheotomy

\begin{tabular}{|c|c|}
\hline Total, $\mathbf{n}$ & 215 \\
\hline Male sex, $\mathrm{n}(\%)$ & $120(55.8)$ \\
\hline Age at tracheotomy, median (IQR), years & $0.8(0.3-4.4)$ \\
\hline$<12$ months, $\mathrm{n}(\%)$ & $127(59.1)$ \\
\hline $1-5$ years, $n(\%)$ & $48(22.3)$ \\
\hline $6-10$ years, $n(\%)$ & $13(6.0)$ \\
\hline $11-15$ years, $n(\%)$ & 27 (12.6) \\
\hline \multicolumn{2}{|l|}{ Indication for tracheotomy } \\
\hline Chronic lung disease, $\mathrm{n}(\%)$ & $79(36.7)$ \\
\hline Neuromuscular disease, $\mathrm{n}(\%)$ & $77(35.8)$ \\
\hline Cardiovascular disease, n (\%) & $53(24.2)$ \\
\hline Upper airway obstruction, n (\%) & $43(20.0)$ \\
\hline Prematurity, n (\%) & $34(15.8)$ \\
\hline Trauma, n (\%) & $16(7.4)$ \\
\hline Prolonged ventilation, n (\%) & $12(5.6)$ \\
\hline Malignancy, n (\%) & $9(4.2)$ \\
\hline Hospital stay, median (Q1; Q3), months & $5.8(2.9 ; 12.2)$ \\
\hline $\begin{array}{l}\text { Mechanical ventilation duration, median } \\
\text { (Q1; Q3), months }\end{array}$ & $9.0(2.0 ; 23.0)$ \\
\hline
\end{tabular}

Duration for tracheotomy dependence, median (Q1; Q3), months

\begin{tabular}{|c|c|}
\hline Discharge with cannulation, $\mathrm{n}(\%)$ & $117(54.4)$ \\
\hline $\begin{array}{l}\text { Never discharged during the study period, } \\
\mathrm{n}(\%)\end{array}$ & $47(21.9)$ \\
\hline Decannulation, n (\%) & $84(39.1)$ \\
\hline $\begin{array}{l}\text { Period from tracheotomy to decannulation, } \\
\text { median (Q1; Q3), months }\end{array}$ & $12.0(1.0 ; 28.3)$ \\
\hline $\begin{array}{l}\text { Complication related to tracheotomy, } \mathrm{n} \\
(\%)\end{array}$ & $45(20.1)$ \\
\hline Mortality, n (\%) & $31(14.4)$ \\
\hline Age at death, median, (IQR), years & $0.7(0.3-7.9)$ \\
\hline
\end{tabular}

months (Q1: 4.1 months; Q3: 34.5 months). One hundred seventeen children $(54.4 \%)$ were discharged with cannulation. During the follow-up period, decannulation was achieved in 84 children $(39.1 \%$ ), and the median time from tracheotomy to decannulation was 12.0 months (Q1: 1.0 month; Q3: 28.3 months) in these children. The number of deaths from all causes was 31 (14.4\%), and the mean age at death was 3.7 years.

\section{Subgroup analysis according to indications}

The age at tracheotomy was different depending on the indications. Tracheotomy was performed later in children with malignancy (median age, 11.0 years, $p<0.0001$ ) than in children without malignancy and later in children with trauma (median age, 8.2 years, $\mathrm{p}<0.0001$ ) than in children without trauma.

The duration of hospitalisation tended to be short in children with prolonged ventilation due to other 
Table 2 Univariate analysis for duration for tracheotomy dependence with or without each disease

\begin{tabular}{llll} 
& \multicolumn{2}{c}{ Duration for tracheotomy dependence, median (IQR), months } & P value \\
\cline { 2 - 3 } & With the disease* & Without the disease* & 0.60 \\
\hline Chronic lung disease & $32.5(4.1-32.5)$ & $15.7(3.6-36.5)$ & 0.0028 \\
Neuromuscular disease & $19.3(12.1-41.6)$ & $11.2(2.0-29.4)$ & 0.42 \\
Cardiovascular disease & $11.2(4.1-31.4)$ & $17.3(4.0-35.5)$ & 0.59 \\
Upper airway obstruction & $18.3(5.1-36.5)$ & $16.7(4.0-33.0)$ & 0.58 \\
Prematurity & $20.3(10.1-28.3)$ & $16.2(4.0-35.5)$ & 0.031 \\
Trauma & $2.5(0.5-26.4)$ & $17.3(5.1-34.5)$ & 0.83 \\
Prolonged ventilation & $22.3(1.0-40.6)$ & $16.2(4.1-33.5)$ & 0.0003 \\
\hline Malignancy & $0(0-2.0)$ & $17.3(4.1-35.5)$ & \\
\hline
\end{tabular}

*With or without the disease showed each left column.

causes (median, 3.9 months) and malignancy (median, 4.0 months), but there was no statistical significance. The duration of mechanical ventilation was longer in children with neuromuscular disease (median, 16.0 months, $\mathrm{p}=0.0011$ ) than in children without neuromuscular disease. Table 2 shows duration for tracheotomy dependence with or without each disease. The duration of tracheotomy dependence was longer in children with neuromuscular disease (median, 19.3 months, $p=0.0028$ ) than in children without neuromuscular disease. Among children with chronic lung disease, neuromuscular disease, cardiovascular disease, upper airway obstruction or premature birth and related conditions, more than half were discharged with cannulation, whereas among children with trauma, prolonged ventilation or malignancy, one-quarter or fewer were discharged with cannulation. Table 3 shows ORs for discharge with cannulation according to the presence of each disease. The rate of discharge with cannulation was significantly higher in children with neuromuscular disease (OR, 2.1; 95\% CI, 1.2 to $3.8, \mathrm{p}=0.047$ ) than in children without neuromuscular disease. Decannulation was achieved in more than half of children with trauma $(62.5 \%)$, prolonged ventilation $(66.7 \%)$ or malignancy $(55.6 \%)$. The rate of

Table 3 ORs for univariate regression analysis of discharge with cannulation according to the presence of each disease

\begin{tabular}{lll}
\hline & $\begin{array}{l}\text { Discharge with } \\
\text { cannulation, OR } \\
\text { (95\% Cl)* }\end{array}$ & P value \\
\hline Chronic lung disease & $1.09(0.62$ to 1.89$)$ & 0.77 \\
\hline Neuromuscular disease & $2.1(1.2$ to 3.8$)$ & 0.047 \\
\hline Cardiovascular disease & $1.19(0.63$ to 2.24$)$ & 0.59 \\
\hline Upper airway obstruction & $1.54(0.77$ to 3.06$)$ & 0.22 \\
\hline Prematurity & $1.24(0.59$ to 2.6$)$ & 0.57 \\
\hline Trauma & $0.25(0.08$ to 0.81$)$ & 0.021 \\
\hline Prolonged ventilation & $0.26(0.07$ to 0.99$)$ & 0.048 \\
\hline Malignancy & $0.097(0.01$ to 0.79$)$ & 0.029 \\
\hline
\end{tabular}

achievement of decannulation was the lowest in children with cardiovascular disease $(30.1 \%)$, but there was no significant difference compared with children without cardiovascular disease. Mortality was significantly higher in children with cardiovascular disease $(\mathrm{OR}, 2.7 ; 95 \% \mathrm{CI}$, 1.2 to $6.0, \mathrm{p}<0.0001)$ than in children without cardiovascular disease.

\section{Complications}

In total, 64 adverse events related to tracheotomy occurred in 45 children $(20.1 \%)$. The most common adverse event was intratracheal granulation $(n=38$, $59.3 \%)$. The first adverse event was most likely to occur 3 months to 1 year after tracheotomy $(\mathrm{n}=19,42.2 \%)$, and only $8.9 \%$ of first adverse events occurred 2 years after the tracheotomy. Adverse events were more likely to occur in patients with tracheotomy dependence for 2 years or more, than in those with tracheotomy dependence for less than 2 years $(\mathrm{n}=27,60.0 \%, \mathrm{p}<0.01)$. Multivariable logistic regression analysis revealed that age was not significantly associated with the occurrence of adverse events, and a 2-year or longer duration of tracheotomy dependence was significantly associated with the occurrence of adverse events (OR, 3.74; 95\% CI, 1.587 to 7.51$)$ (table 4).

Table 4 Multivariable logistic regression analysis of risk factors for occurrence of complications

\begin{tabular}{|c|c|c|}
\hline & OR, $95 \% \mathrm{Cl}$ & P value \\
\hline \multicolumn{3}{|l|}{ Age } \\
\hline$<12$ months & 1 (reference) & \\
\hline $1-5$ years & $1.76(0.80$ to 3.90$)$ & 0.11 \\
\hline $6-10$ years & 0.89 (0.18 to 4.47$)$ & 0.84 \\
\hline $11-15$ years & 0.65 (0.17 to 2.40$)$ & 0.40 \\
\hline \multicolumn{3}{|c|}{ Duration for tracheotomy dependence } \\
\hline$<2$ years & 1 (reference) & \\
\hline$\geq 2$ years & $3.74(1.87$ to 7.1$)$ & 0.0002 \\
\hline
\end{tabular}




\section{DISCUSSION}

In this descriptive study, we investigated the incidence, indications and outcomes of paediatric tracheotomy in Japan, such as the length of hospital stay, weaning from ventilation, complications, decannulation and mortality.

\section{Age and indications}

As in recent studies, we found that tracheotomies were performed most often when patients were less than 12 months old $(\mathrm{n}=127,59.1 \%) .{ }^{5-813}$ In the premature birth and related conditions and neuromuscular disease groups in our study, most tracheotomies were performed within the first year of life, possibly due to the high rate of comorbidities among preterm infants who survived beyond the neonatal period. ${ }^{14}$

We accounted for children with multiple indications for tracheotomy because children with a chronic medical condition might have two or more comorbidities. In our study, $44.7 \%$ of children who underwent tracheotomy had two or more indications, and this rate was $60 \%$ in a previous study. ${ }^{5}$

Table 5 summarises the indications for tracheotomy from previous studies after 1997. Table 5 shows some representative studies reporting indications and outcomes of paediatric tracheotomy over the most recent two decades. The frequency of each indication varied depending on the study, possibly because the method of classifying indications varied, and some studies allowed children to have multiple indications while others did not. In our study, chronic lung disease was the most common indication for tracheotomy, similar to previous studies that also allowed children to have multiple indications for tracheotomy. ${ }^{5713}$ The most commonly paired indications were chronic lung disease and neuromuscular disease, consistent with one of the previous studies. ${ }^{5}$ Due to respiratory muscle weakness, recurrent aspiration, poor cough and airway clearance, and chest wall deformity by kyphoscoliosis, neuromuscular disease increases the risk of respiratory difficulties. ${ }^{15}$ When evaluating the frequency for each indication, chronic lung disease was the most common indication for tracheotomy, followed by neuromuscular disease, which might be a consequence of the increasing survival of children with conditions such as neonatal intracranial haemorrhage, encephalitis and hypoxic encephalopathy. The second most commonly paired indications were chronic lung disease and premature birth. This could be because premature infants have complex and severe physical disabilities, and children with such disabilities often have respiratory problems.

Upper airway obstruction was the fourth most common indication for tracheostomy in our study. Among the causes of upper airway obstruction, congenital malformations were the main indications for tracheotomy, and only a few tracheotomies were performed for infections in our study and in previous studies, ${ }^{8911}$ probably due to the widespread use of vaccination, such as the $\mathrm{H}$. influenzae type $\mathrm{B}$ and pneumococcal conjugate vaccines, and the decrease in the occurrence of severe infection. ${ }^{4}$

\section{Cannulation and decannulation}

The duration of tracheotomy dependence and the rate of decannulation vary depending on the underlying diseases. ${ }^{10}$ In patients who had trauma, the duration of tracheotomy dependence was relatively shorter, and the rate of decannulation was higher than in congenital or chronic disease patients, which was consistent with the findings of previous studies. ${ }^{8-10}$ The rate of decannulation has decreased over time. ${ }^{1}$ The rate of decannulation varies between studies from $8.8 \%$ to $42.0 \%$, even when only comparing studies conducted after $1997 .{ }^{15810}$ The overall rate of decannulation was $39.1 \%$ in our study which was within the range of that from previous studies. The variation in the decannulation rate between studies may be related to patient characteristics, especially the underlying disease and follow-up periods. In our study, we used a nationwide insurance claims database that tracked data for each patient, even if the patient visited or was hospitalised at multiple medical institutions. The use of this database helped us to prevent underestimating the rate of decannulation missed during follow-up when patients were transferred to other hospitals.

\section{Complications related to tracheotomy and mortality}

As in previous studies, the most common complication related to tracheotomy in this study was intratracheal granulation. ${ }^{89}$ Adverse events were more likely to occur in patients with tracheotomy dependence for 2 years or more, whereas in most patients, the first adverse event occurred within 2 years after tracheotomy. Children who require long-term tracheotomy may tend to have complications due to a poor general condition such as malnutrition, immunocompromise, bleeding tendency and metabolic disorders.

In our study, cardiovascular disease was associated with higher mortality, similar to a previous study. ${ }^{6}$ In this previous study, Berry et al concluded that congenital heart disease, prematurity, absence of an upper airway anomaly and age $<1$ year were characteristics associated with higher mortality.

\section{Limitations}

There were several limitations of this study that should be considered. First, the database used in this study was collected for billing purposes, and we could not obtain detailed clinical information for certain conditions. We estimated the indications for tracheotomy from the disease codes, but we could not determine the severity and detailed conditions of the diseases. Furthermore, it was difficult to make precise comparisons across the literature due to varying classifications for indications. Second, in some patients, we may have underestimated the durations of mechanical ventilation and tracheotomy dependence as they may have continued after the follow-up period of this study. Third, adverse events without diagnosis codes, such as accidental decannulation, could not be identified. The complication rate was $20.1 \%$ in our study, which was lower than those of previous studies, which range 


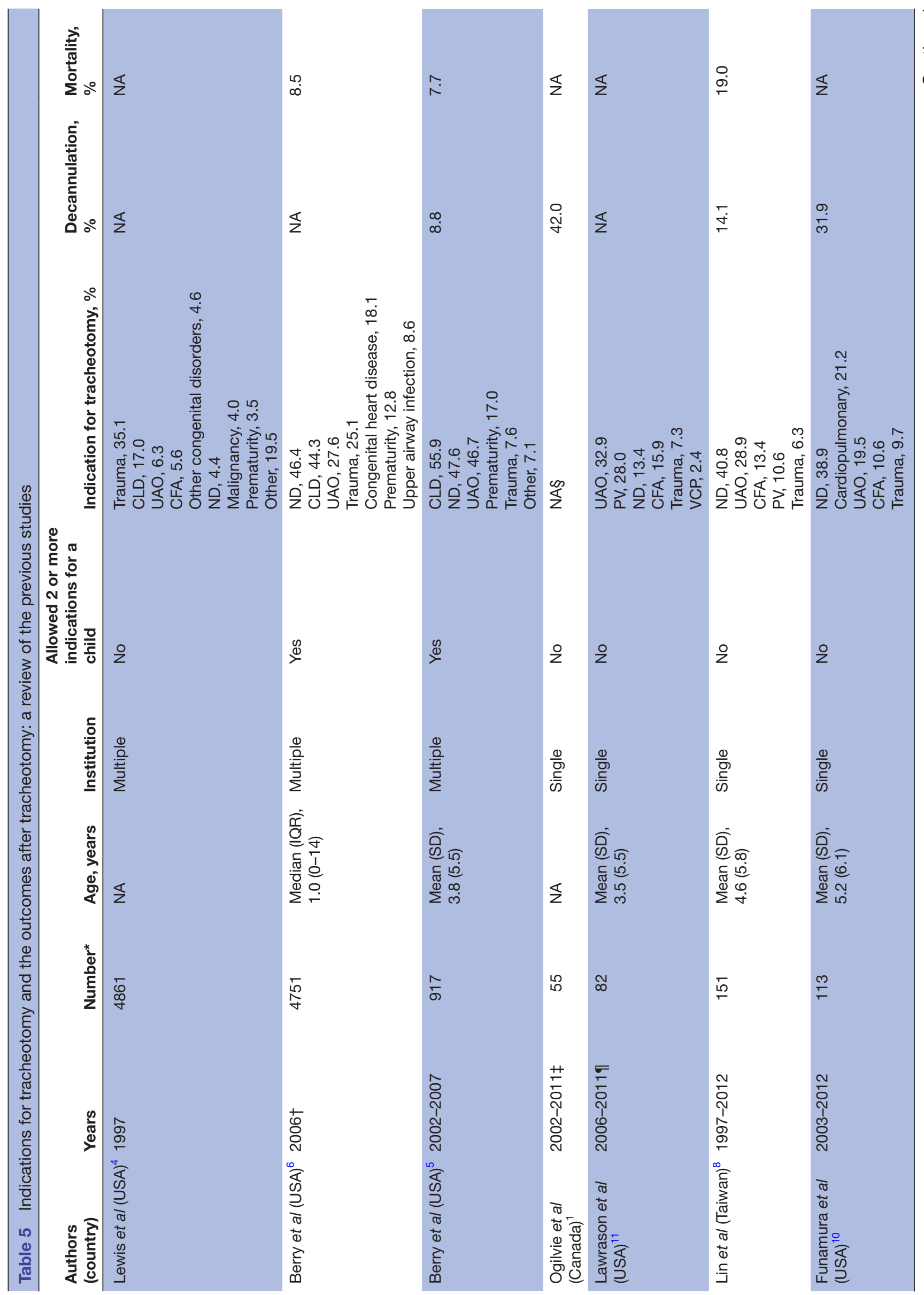



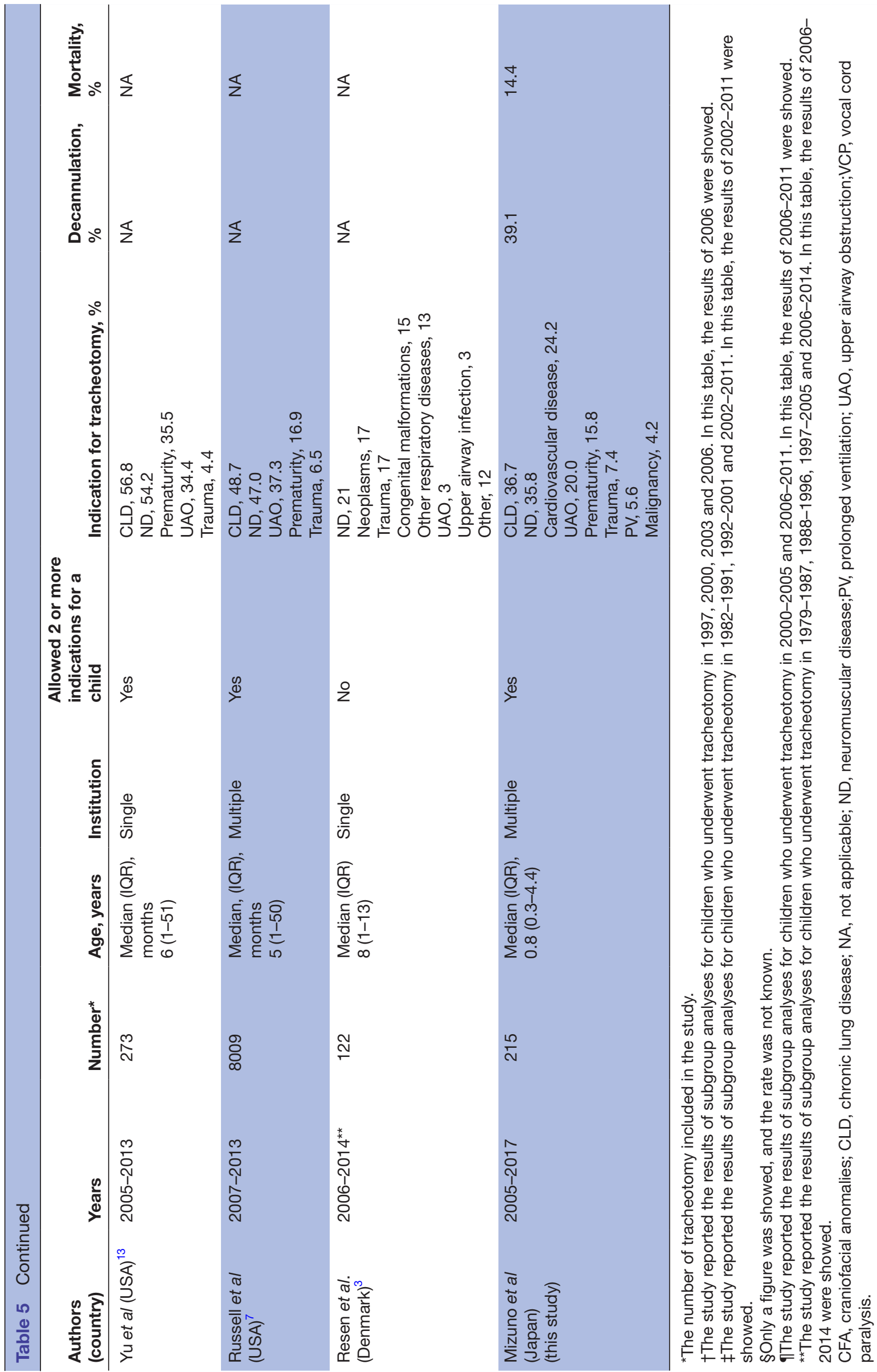
from $22 \%-43 \% .{ }^{189}$ Fourth, the cause of death could not be identified from the database. According to previous studies, the tracheostomy-related mortality rates ranged from $0 \%$ to $4 \%$, and the most common cause was accidental decannulation. ${ }^{19}$ The overall mortality rate was $14.4 \%$ in our study, similar to the $15 \%-20 \%$ mortality rates observed in previous studies. ${ }^{19}$ Fifth, the JMDC database may not reflect the population of all paediatric patients with tracheostomy in Japan because the coverage rate of the database was $3 \%$ of the Japanese population. However, we assume that the characteristics of our patient population were similar to those of the whole paediatric patient population with tracheotomy in Japan. The first reason for this assumption is the low medical cost for caregivers. In most regions in Japan, medical expenses for children are largely covered by public support and are virtually free for parents. This situation allows physicians to deliver universal care to children if medically indicated, regardless of insurance type or, caregivers' socioeconomic status or income. Second, access to a medical institution is relatively easy, and the waiting list is not very long, even in specialised centres. For these reasons, children in Japan can adequately receive medical care in a timely manner, irrespective of their insurance type or caregivers' socioeconomic status.

In summary, this descriptive study using a nationwide insurance claims database demonstrated that paediatric tracheotomies in Japan were performed most often when patients were younger than 12 months, and the most common indication for tracheotomy was chronic lung disease. This study adds to the existing literature by providing an analysis of children who underwent tracheotomy tracked across multiple hospitals and is the first multi-institutional analysis in an Asian country. In patients with chronic lung disease, neuromuscular disease, upper airway obstruction or cardiovascular disease, more than half were discharged with cannulation, and the duration of tracheotomy dependence was longer and the decannulation rate was lower than in patients who had trauma. The long-term duration of tracheotomy dependence might have some impact on patients' physical and mental development and the quality of life of the patients and their families. Further research should be necessary to investigate further changes in the indications for paediatric tracheotomy, the prognosis after tracheotomy and the quality of life of patients and their families.

Contributors KM designed the study and wrote the initial draft of the manuscript. MT contributed to the analysis and interpretation of the data and assisted in the preparation of the manuscript. YK, KK and KO contributed to data interpretation and critically reviewed the manuscript. All authors approved the final version of the manuscript and agree to be accountable for all aspects of the work in ensuring that questions related to the accuracy or integrity of any part of the work are appropriately investigated and resolved.

Funding The authors have not declared a specific grant for this research from any funding agency in the public, commercial or not-for-profit sectors.

Competing interests None declared.

Patient consent for publication Not required.

Ethics approval This study was approved by the Kyoto University Graduate School and Faculty of Medicine Ethics Committee (R1539).

Provenance and peer review Not commissioned; externally peer reviewed.

Data availability statement № data are available.

Open access This is an open access article distributed in accordance with the Creative Commons Attribution Non Commercial (CC BY-NC 4.0) license, which permits others to distribute, remix, adapt, build upon this work non-commercially, and license their derivative works on different terms, provided the original work is properly cited, appropriate credit is given, any changes made indicated, and the use is non-commercial. See: http://creativecommons.org/licenses/by-nc/4.0/.

ORCID iD

Koji Kawakami http://orcid.org/0000-0002-7477-4071

\section{REFERENCES}

1 Ogilvie LN, Kozak JK, Chiu S, et al. Changes in pediatric tracheostomy 1982-2011: a Canadian tertiary children's Hospital review. J Pediatr Surg 2014;49:1549-53.

$2 \mathrm{Ha} \mathrm{T-A}$, Goyal M, Ongkasuwan J. Duration of tracheostomy dependence and development of tracheocutaneous fistula in children. Laryngoscope 2017;127:2709-12.

3 Resen MS, Grønhøj C, Hjuler T. National changes in pediatric tracheotomy epidemiology during 36 years. Eur Arch Otorhinolaryngol 2018;275:803-8.

4 Lewis CW, Carron JD, Perkins JA, et al. Tracheotomy in pediatric patients: a national perspective. Arch Otolaryngol Head Neck Surg 2003;129:523-9.

5 Berry JG, Graham DA, Graham RJ, et al. Predictors of clinical outcomes and hospital resource use of children after tracheotomy. Pediatrics 2009;124:563-72.

6 Berry JG, Graham RJ, Roberson DW, et al. Patient characteristics associated with in-hospital mortality in children following tracheotomy. Arch Dis Child 2010;95:703-10.

7 Russell CJ, Thurm C, Hall M, et al. Risk factors for hospitalizations due to bacterial respiratory tract infections after tracheotomy. Pediatr Pulmonol 2018;53:349-57.

8 Lin C-Y, Ting T-T, Hsiao T-Y, et al. Pediatric tracheotomy: a comparison of outcomes and lengths of hospitalization between different indications. Int J Pediatr Otorhinolaryngol 2017;101:75-80.

9 de Trey L, Niedermann E, Ghelfi D, et al. Pediatric tracheotomy: a 30-year experience. J Pediatr Surg 2013;48:1470-5.

10 Funamura JL, Durbin-Johnson B, Tollefson TT, et al. Pediatric tracheotomy: indications and decannulation outcomes. Laryngoscope 2014;124:1952-8.

11 Lawrason A, Kavanagh K. Pediatric tracheotomy: are the indications changing? Int J Pediatr Otorhinolaryngol 2013;77:922-5.

12 Kimura S, Sato T, Ikeda S, et al. Development of a database of health insurance claims: standardization of disease classifications and anonymous record linkage. J Epidemiol 2010;20:413-9.

$13 \mathrm{Yu} \mathrm{H}$, Mamey MR, Russell CJ. Factors associated with 30-day allcause Hospital readmission after tracheotomy in pediatric patients. Int J Pediatr Otorhinolaryngol 2017;103:137-41.

14 Shah PS, Lui K, Sjörs G, et al. Neonatal outcomes of very low birth weight and very preterm neonates: an international comparison. $J$ Pediatr 2016;177:144-52.

15 Seddon PC, Khan Y. Respiratory problems in children with neurological impairment. Arch Dis Child 2003;88:75-8. 